\title{
Proceso de Gestión de Asistencia Técnica. Caso de estudio: Hospital General Isidro Ayora de Loja, Ecuador
}
Technical Assistance Management Process. Case study: General Hospital Isidro Ayora de Loja, Ecuador

\section{Processo de Gestão de Assistência Técnica. Estudo de caso: Hospital Geral Isidro Ayora de Loja, Equador}

\author{
Germán David Salas-Paucar ${ }^{\text {I }}$ \\ Dvicho88@gmail.com \\ Milton Alfredo Campoverde-Molina II \\ mcampoverde@ucacue.edu.ec
}

Recibido: 10 de mayo de 2019 *Aceptado: 18 de junio de 2019 * Publicado: 05 de julio de 2019

I. Ingeniero de Sistemas, Jefatura de Posgrados, Universidad Católica de Cuenca, Cuenca, Ecuador.

I. Ingeniero en Sistemas, Docente de la Unidad Académica de Tecnologías de la Información, Jefatura de Posgrados, Universidad Católica de Cuenca, Cuenca, Ecuador. 


\section{Resumen}

Este artículo presenta la propuesta del Proceso de Gestión de Asistencia Técnica (PGAT) en el departamento de Tecnologías de la Información y Comunicaciones (TIC's) del Hospital Isidro Ayora de Loja, Ecuador. El propósito de la investigación es mediante el uso de la metodología de Gestión de Procesos de Negocios (BPM), levantar, documentar, diseñar, ejecutar y analizar el PGAT. Se revisaron diferentes fuentes bibliografías referentes a experiencias y resultados de investigaciones de gestión de procesos de negocios en el sector salud. Se analizó información correspondiente asistencia técnica brindada durante el año 2018 en el departamento de TIC's, en total 2.777 asistencias. Con los datos obtenidos se realizó el modelado del proceso de acuerdo a la metodología BPM, para lo cual se hizo uso de la herramienta Bizagi Modeler en la que se diseñó y simulo el proceso. Los resultados obtenidos de la simulación permitieron determinar que la eficiencia del proceso PGAT disminuía el tiempo de atención en un 46.3\% en relación al tiempo necesario para atender la misma cantidad de incidencias de forma manual. Se concluyó que la automatización de este proceso reduciría el tiempo empleado en resolver las solicitudes de asistencia generadas por los usuarios y mejorara sustancialmente el grado de cumplimiento de la gestión de asistencia técnica brindada.

Palabras clave: Tecnología; hospital; BPM; BPMN; Bizagi.

\section{Abstract}

This article presents the proposal of the Technical Assistance Management Process (PGAT) in the Department of Information and Communication Technologies (TIC's) of the Isidro Ayora Hospital in Loja, Ecuador. The purpose of the research is to use the methodology of Business Process Management (BPM), raise, document, design, execute and analyze the PGAT. Different sources bibliographies referring to experiences and research results of business process management in the health sector were reviewed. The corresponding technical assistance information was analyzed during the year 2018 in the TIC department, in total 2,777 assists. With the data obtained, the modeling of the process was carried out according to the BPM methodology, for which use was made of the Bizagi Modeler tool in which the process was designed and simulated. The results obtained from the simulation allowed to determine that the efficiency of the PGAT process decreased the attention time by $46.3 \%$ in relation to the time necessary to attend the same number

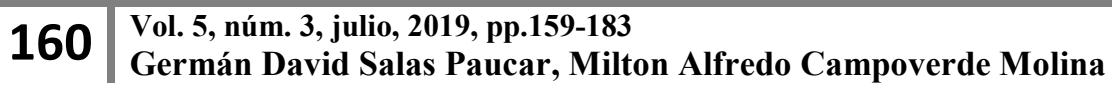


of incidences manually. It was concluded that the automation of this process would reduce the time spent in resolving the requests for assistance generated by the users and would substantially improve the degree of compliance with the technical assistance management provided.

Key words: Technology; hospital; BPM; BPMN; Bizagi.

\section{Resumo}

O Este artigo apresenta a proposta do Processo de Gestão de Assistência Técnica (PGAT) no Departamento de Tecnologias de Informação e Comunicação (TIC's) do Hospital Isidro Ayora em Loja, Equador. O objetivo da pesquisa é utilizar a metodologia de Business Process Management (BPM), levantar, documentar, projetar, executar e analisar o PGAT. Diferentes fontes bibliográficas referentes a experiências e resultados de pesquisas de gestão de processos de negócios no setor saúde foram revisadas. A informação de assistência técnica correspondente foi analisada durante o ano de 2018 no departamento de TIC, totalizando 2.777 assistências. Com os dados obtidos, a modelagem do processo foi realizada de acordo com a metodologia BPM, para a qual foi feito uso da ferramenta Bizagi Modeler, na qual o processo foi projetado e simulado. Os resultados obtidos a partir da simulação permitiram determinar que a eficiência do processo PGAT diminuiu o tempo de atendimento em $46,3 \%$ em relação ao tempo necessário para atender ao mesmo número de incidências manualmente. Concluiu-se que a automação desse processo reduziria o tempo despendido na resolução dos pedidos de assistência gerados pelos usuários e melhoraria substancialmente o grau de cumprimento da gestão da assistência técnica prestada.

Palavras-chave: Tecnologia; hospital; BPM; BPMN; Bizagi.

\section{Introducción}

La Organización Mundial de la Salud (OMS) es el organismo de la Naciones Unidas, responsable de la salud a nivel mundial. Es el encargado de elaborar directrices y normas sanitarias además de promover la investigación en el sector salud (EcuRed, 2018). Para las Américas el organismo encargado de la salud pública es la Organización Panamericana de la Salud (OPS) (Organización Panamericana de la Salud, 2014). En el Ecuador el Ministerio de Salud Pública (MSP) es el encargado de velar por la salud pública (Ministerio de Salud Pública, 2012d). El MSP tiene como misión "Ejercer la rectoría, regulación, planificación, coordinación, control y gestión de la Salud Pública ecuatoriana a través de la gobernanza, vigilancia y control sanitario además garantiza el 
derecho a la Salud a través de la provisión de servicios de atención individual, prevención de enfermedades, promoción de la salud, la gobernanza de salud, investigación y desarrollo de la ciencia y tecnología, articulando los actores del sistema con el fin de garantizar el derecho a la Salud" (Ministerio de Salud Pública, 2012c). Para cumplir con esta misión el MSP mediante acuerdo ministerial No. 00001537 con fecha de publicación 12 de octubre de 2012, emite el Estatuto Orgánico de Gestión Organizacional por Procesos de los Hospitales del Ministerio de Salud Pública, que rige de acuerdo a la constitución de la República del Ecuador (Ministerio de Salud Pública, 2012b).

De acuerdo a este estatuto (MSP, 2012), los Hospitales del MSP desarrollan los procesos internos que se clasifican en función de su grado de contribución o valor agregado al cumplimiento de su misión. Por consiguiente, para los Hospitales Generales se ordenan y clasifican de la siguiente manera:

Proceso Gobernante: Direccionamiento Estratégico del Hospital (Gerencia)

Procesos Agregadores de Valor: Gestión Asistencial

Procesos Habilitantes de Asesoría: Gestión de Planificación, Seguimiento y Evaluación de Gestión, Gestión de Asesoría Jurídica, Gestión de Comunicación y Gestión de Calidad

Procesos Habilitantes de Apoyo: Gestión de Atención al Usuario, Gestión de Admisiones, Gestión Administrativa y Financiera.

De acuerdo a las "Normas de control interno para las entidades, organismos del sector público y de las personas jurídicas de derecho privado que dispongan de recursos públicos" de la Contraloría General del Estado (CGE) (Contraloría General del Estado, 2009), "Las entidades y organismos del sector público deben estar acopladas en un marco de trabajo para procesos de tecnología de información que aseguren la transparencia y el control, así como el involucramiento de la alta dirección, por lo que las actividades y procesos de tecnología de información de la organización deben estar bajo la responsabilidad de una unidad que se encargue de regular y estandarizar los temas tecnológicos a nivel institucional".

\footnotetext{
\begin{tabular}{l|l}
162 & Vol. 5, núm. 3, julio, 2019, pp.159-183 \\
Germán David Salas Paucar, Milton Alfredo Campoverde Molina
\end{tabular}
} 
El Hospital General Isidro Ayora de Loja (HIAL) pertenece a la Coordinación Zonal 7 - Salud (Ministerio de Salud Pública, 2015a). Orienta sus servicios a la atención social destinando la mayor cantidad de recursos al equipamiento y adquisición de servicios dirigidos a este entorno. El HIAL cuenta con el manual de procesos operativos, en el cual se establecen los procesos encargados de generar y administrar los productos y servicios destinados a los usuarios y permiten cumplir con la misión institucional y los objetivos estratégicos (Ministerio de Salud Pública, 2016). En vista de la importancia que tiene un manual de procesos para contribuir con la misión institucional y los objetivos estratégicos, es evidente la necesidad de contar con un manual de procesos que abarque todas las áreas de la parte administrativa del HIAL, de tal manera que los funcionarios de estas áreas conozcan cómo están estructurados los procesos que ahí se ejecutan.

El departamento de Tecnologías de la Información y Comunicaciones juega un papel muy importante dentro de los Hospitales del MSP, es así que tiene como misión "Aplicar las normas y procedimientos que efectivicen la gestión y administración de las tecnologías de la información y comunicaciones, orientadas a la optimización de los recursos y fortalecimiento de la red interna para mejorar la eficiencia en la atención a los pacientes" (Ministerio de Salud Pública, 2012b). Para cumplir con esta misión es importante contar con procesos ya definidos que permitan cumplir de manera adecuada con los productos y servicios de esta unidad. También es importante que el personal de la institución conozca que sus actividades individuales forman parte de estos procesos y que estos contribuyen a alcanzar los objetivos estratégicos de la institución.

El departamento de Tecnologías de la Información y Comunicaciones del HIAL, se ubica dentro de los Procesos Habilitantes de Asesoría y de Apoyo de acuerdo a la estructura orgánica de Hospitales Generales del MSP. Funciona de acuerdo a políticas, normas y procedimientos establecidos desde planta central por la Dirección Nacional de Tecnologías de la Información y Comunicaciones (DNTICS) (Ministerio de Salud Pública, 2015b). Dentro de estas normas y políticas se encuentra la Política Coordinación del Centro de Soporte de Tecnología, la cual entre sus responsabilidades tiene: gestionar las peticiones de servicios de los usuarios (Ministerio de Salud Pública, 2012a). Para atender estas peticiones el departamento de TIC's elaboro un Acuerdo de Nivel de Servicio (SLA) el cual es tomado como referencia para los Hospitales Generales de la

\footnotetext{
\begin{tabular}{l|l}
163 & Vol. 5, núm. 3, julio, 2019, pp.159-183 \\
Germán David Salas Paucar, Milton Alfredo Campoverde Molina
\end{tabular}
} 
Zona 7 - Salud. Tiene como objetivo recoger los términos y condiciones para la prestación de servicios establecidos entre el departamento de TIC's y los usuarios. Además, en el SLA se determina el método de resolución de los incidentes o requerimientos solicitados por los usuarios internos (Hospital General Isidro Ayora, 2017). El proceso de resolución de asistencia técnica es uno de los más complejos dentro del departamento de TIC's ya que no brindar un adecuado soporte a las solicitudes reportadas por los usuarios dificulta el cumplimiento de sus actividades y por ende el cumplimiento de la visión institucional.

El propósito de esta investigación es analizar las solicitudes de asistencia técnica del departamento de TIC's del HIAL durante el año 2018 y proponer un proceso mediante la utilización de herramienta de diagramación bajo estándar BPMN, con lo cual se busca dar respuesta a las siguientes preguntas:

- ¿Existen procesos definidos para la gestión de asistencia técnica del departamento de Tecnologías de la Información del Hospital Isidro Ayora de Loja?

- ¿La implementación de una metodología BPMN (Business Process Model and Notation) mejorará la gestión de asistencias técnicas en el Departamento de TIC's del Hospital Isidro Ayora de Loja?

En cuanto a la estructura y contenido del artículo, en la sección 2 se presentan los conceptos relacionados con la investigación. En la sección 3, la revisión de diferentes fuentes bibliográficas de experiencias y resultados de investigaciones de gestión de procesos de negocios. En la sección 4, se detalla la metodología utilizada para desarrollar esta investigación. En la sección 5, se muestran los resultados del análisis de las asistencias técnicas y la propuesta de BPMN en el departamento de TIC's del HIAL. En la sección 6, se establecen las conclusiones de acuerdo a los resultados obtenidos y en la sección 7 se describen posibles trabajos futuros a partir de esta investigación. 


\section{Desarrollo}

\section{Business Process Management (BPM)}

Orienta los esfuerzos para la optimización de los procesos de la empresa mediante el modelado, automatización, ejecución, control, medición y optimización de los flujos de actividades de negocio para conseguir los objetivos de la empresa, integrando personas, sistemas, funciones, negocios, clientes, proveedores y socios dentro y fuera de los límites de la empresa (Leon, 2010). Además, permite integrar el recurso humano con las TIC's para fomentar procesos de negocio efectivos, ágiles y transparentes (Garimella, Michael Lees, \& Bruce Williams, 2012).

\section{Business Process Model and Notation BPMN}

El flujo de actividades que conforman los procesos de negocio de una empresa, se representan mediante la notación grafica estandarizada Business Process Model and Notation (BPMN), en español Modelo y Notación de Procesos de Negocio. Esta notación gráfica plasma en un diagrama la secuencia de actividades, los mensajes entre los diferentes participantes y toda la información que es necesaria para que un proceso sea analizado, simulado y ejecutado (Bizagi, 2003).

Tabla 1: Notación básica BPMN

\begin{tabular}{|l|l|}
\hline \multicolumn{1}{|c|}{ Elemento } & \multicolumn{1}{|c|}{ Descripción } \\
\hline \multicolumn{1}{|c|}{ Tareas } \\
\hline Tarea & $\begin{array}{l}\text { Es una actividad atómica dentro de un flujo de } \\
\text { proceso. }\end{array}$ \\
\hline \multicolumn{3}{|c|}{ Subprocesos } \\
\hline $\begin{array}{l}\text { Subproceso } \\
\text { Reusable }\end{array}$ & $\begin{array}{l}\text { Identifica un punto en el flujo donde se invoca un } \\
\text { proceso pre-definido. }\end{array}$ \\
\hline \multicolumn{1}{|c|}{ Compuertas } \\
\hline $\begin{array}{l}\text { Compuerta } \\
\text { Exclusiva }\end{array}$ & $\begin{array}{l}\text { De divergencia: Se utiliza para crear caminos } \\
\text { alternativos } \\
\text { De convergencia: Se utiliza para unir caminos } \\
\text { alternativos. }\end{array}$ \\
\hline $\begin{array}{l}\text { Compuerta } \\
\text { Inclusiva }\end{array}$ & $\begin{array}{l}\text { De divergencia: al menos un camino debe ser } \\
\text { activado para continuar con el flujo del proceso. } \\
\text { De convergencia: más de un camino puede llegar a } \\
\text { ella, pero solo puede tener un camino de salida }\end{array}$ \\
\hline
\end{tabular}


Dom. Cien., ISSN: 2477-8818

Vol. 5, núm. 3, julio, 2019, pp. 159-183

Proceso de Gestión de Asistencia Técnica. Caso de estudio: Hospital General Isidro Ayora de Loja, Ecuador

\begin{tabular}{|c|c|c|}
\hline \multicolumn{3}{|c|}{$\begin{array}{l}\text { Eventos de inicio } \\
\end{array}$} \\
\hline $\begin{array}{l}\text { Evento de Inicio } \\
\text { Simple }\end{array}$ & $\begin{array}{l}\text { Indica dónde se inicia un proceso. No tiene algún } \\
\text { comportamiento particular. }\end{array}$ & \\
\hline $\begin{array}{l}\text { Evento de Inicio } \\
\text { de Mensaje }\end{array}$ & $\begin{array}{l}\text { Se utiliza cuando el inicio de un proceso se da al } \\
\text { recibir un mensaje de un participante externo. }\end{array}$ & \\
\hline \multicolumn{3}{|c|}{ Eventos Intermedios } \\
\hline $\begin{array}{l}\text { Evento } \\
\text { Intermedio } \\
\text { Simple }\end{array}$ & $\begin{array}{l}\text { Indica que algo sucede en algún lugar entre el inicio } \\
\text { y el final de un proceso. }\end{array}$ & \\
\hline $\begin{array}{l}\text { Evento de } \\
\text { Mensaje }\end{array}$ & $\begin{array}{l}\text { Indica que un mensaje puede ser enviado o recibido. } \\
\text { Si un proceso está esperando un mensaje y éste es } \\
\text { capturado, el proceso continuará su flujo. El } \\
\text { marcador de eventos en esta instancia estará lleno. } \\
\text { El evento que lanza un mensaje se identifica con una } \\
\text { figura sombreada. El evento que capta un mensaje se } \\
\text { identifica con una figura sin relleno. }\end{array}$ & $\begin{array}{l}\text { Message } \\
\text { Throw } \\
\text { Message } \\
\text { Catch }\end{array}$ \\
\hline Evento de Enlace & $\begin{array}{l}\text { Este evento permite conectar dos secciones del } \\
\text { proceso si se encuentran muy separadas. }\end{array}$ & (ink Thro... Link Catch \\
\hline \multicolumn{3}{|c|}{ Eventos de Finalización } \\
\hline $\begin{array}{l}\text { Finalización } \\
\text { simple }\end{array}$ & Indica que el flujo finaliza. & \\
\hline \multicolumn{3}{|c|}{ Carriles (Swim lanes) } \\
\hline $\begin{array}{l}\text { Contenedor } \\
\text { (Pool) }\end{array}$ & Un pool es un contenedor de procesos simples. & \\
\hline Carril (Lane) & $\begin{array}{l}\text { Es una sub-partición dentro del proceso. Se utilizan } \\
\text { para diferenciar roles internos, posiciones, } \\
\text { departamentos. }\end{array}$ & i: \\
\hline \multicolumn{3}{|c|}{ Conectores } \\
\hline $\begin{array}{l}\text { Flujo de } \\
\text { Secuencia }\end{array}$ & $\begin{array}{l}\text { Muestra el orden en el que las actividades se } \\
\text { ejecutarán }\end{array}$ & \\
\hline Flujo de Mensaje & $\begin{array}{l}\text { Se utiliza para mostrar el flujo de mensajes entre } \\
\text { dos entidades. }\end{array}$ & \\
\hline
\end{tabular}

Fuente: (Bizagi, 2003). 


\section{Herramienta para gestión de procesos}

La importancia del análisis, modelado y gestión de un proceso de negocios no se limita a un sector empresarial específico (Rolón, Chavira, Orozco, \& Soto, 2015). Las herramientas de gestión de procesos son suite BPM que ayudan a gestionar el ciclo de vida completo de procesos. Deben contar con dos elementos esenciales: un entorno de modelado y simulación de procesos, y un motor de ejecución de procesos.

\section{Bizagi}

Es una suite que contiene productos complementarios, Modelador de Procesos y una Suite de BPM. Contiene una plataforma de automatización de procesos diseñada para apoyar la transformación empresarial. Bizagi reduce el "time-to-market" de nuevas ideas y estrategias de negocio, y facilita el mejoramiento continuo de sus procesos (Bizagi, 2014).

\section{Bizagi BPM suite}

Maneja el ciclo de vida completo de un proceso de negocio: Modelar, Construir y Ejecutar. Cada una de estos pasos se realiza en distintas soluciones que permiten, a través de un ambiente gráfico y dinámico, construir una solución basada en procesos (Bizagi, 2014).

\section{Bizagi Process Modeler}

Bizagi Modeler es una aplicación que le permite modelar visualmente, documentar, simular, publicar, y compartir procesos de negocio bajo un estándar de la industria como lo es BPMN (Bizagi, 2016).

\section{Trabajos relacionados}

En una investigación desarrollada en el 2015, se propuso el diseño de un proceso de negocio para la planificación de sistemas de información de empresas de la industria biofarmacéutica cubana. Como resultado se determinó que: mediante el diseño de un proceso, se definen registros que facilitan la diferenciación de sistemas de información, de sistemas informáticos. El proceso de planificación propuesto permite ajustar la evolución de los sistemas de información a las 
necesidades de la organización, en función del logro de sus objetivos estratégicos. Este resultado se combina con el modelado de procesos utilizando la notación BPM; se flexibiliza el modelado y se facilita la comprensión y la automatización del proceso (Hernández, Pérez, León, Infante, \& Blanco, 2015).

En un estudio presentado en el 2015, se propone ampliar y adaptar un lenguaje de modelado de procesos como BPMN y enriquecer este lenguaje con capacidades para un modelado empresarial integral que cubra perspectivas y diagramas. Como resultado se presenta la arquitectura y el diseño de una extensión revisada de BPMN para el modelado de múltiples perspectivas, y la aplicabilidad de la extensión propuesta se demuestra al modelar una parte $\mathrm{CP}$ (vías clínicas) para el tratamiento de pacientes con accidente cerebrovascular, que integra explícitamente el proceso y la perspectiva de recursos (Braun, Burwitz, Schlieter, \& Benedict, 2015).

En el año 2015, se realizó un estudio en un hospital en Lisboa, Portugal, en el cual se presenta y discute los resultados en términos de complejidad de la introducción de un nuevo sistema de programación dentro de los procesos comerciales de exámenes y citas médicas. Durante el estudio de caso se modeló los procesos utilizando el estándar BPMN. Además, utilizando métricas abstractas se comparó la complejidad entre los procesos antiguos y los nuevos. Como resultado se concluyó que los nuevos procesos de negocios prácticamente permanecían como estaban y que el nuevo sistema de programación no trajo una reducción significativa en la complejidad de los procesos, teniendo en cuenta el número de actividades, elementos de control-flujo, y la complejidad global de control-flujo (Martinho, Rijo, \& Nunes, 2015).

En el año 2015, se desarrolló un trabajo de investigación mediante la aplicación del modelado de procesos de negocio al sector salud, con el objetivo de mostrar la experiencia obtenida en la creación de modelos conceptuales de ciertos procesos hospitalarios que se puedan usar como base para otros en colaboración con los hospitales para modelar sus procesos utilizando BPMN. Como resultado se pudo conocer la utilidad de los modelos para administrar estos procesos, además proponerles mejoras, identificando modelos que sean más fáciles de entender y modificar (Rolón et al., 2015). 
En una investigación presentada en el año 2017, se realizó una propuesta de gestión de procesos de negocios para desarrollar una plataforma informática que de soporte a la gestión de servicios académicos (GSA), como a las Prácticas Pre-profesionales (PPP) en el ámbito universitario. Como resultado se determinó que Bizagi Studio fue la tecnología apropiada por su funcionalidad, escalabilidad y capacidad de integración. Además, se ha mostrado que es posible mejorar sustancialmente el grado de cumplimiento de objetivos funcionales en la gestión de procesos (Congacha \& Garcia, 2017).

\section{Metodología}

A continuación, se describe el procedimiento utilizado para el desarrollo de la presente investigación:

\section{Recolección y Análisis de información de Asistencias Téenicas (PGAT): se utilizó los} reportes de asistencia técnicas brindadas por el departamento de TIC's del Hospital Isidro Ayora de la ciudad de Loja durante el 2018. Se seleccionó la información necesaria para el levantamiento del proceso mediante el análisis de 2.777 asistencias registradas por el personal del Departamento de Tecnologías de la Información y Comunicaciones del Hospital Isidro Ayora de Loja, Ecuador.

2. Modelado del Proceso de Gestión de Asistencia Técnica (PGAT): de acuerdo al análisis de asistencias técnicas, y en base al SLA del departamento de TIC's, se realizó el diseño y diagramación del proceso denominado "Proceso de Gestión de Asistencia Técnica" (PGAT). Para esto se utilizó la herramienta de diagramación Bizagi Modeler en el cual se plasmó las tareas, los eventos intermedios, eventos de inicio, eventos de finalización, los caminos que debe seguir y sus secuencias.

3. Validación del Proceso de Gestión de Asistencia Técnica: para validar el proceso en los diferentes escenarios se utilizó la herramienta Bizagi, en la cual luego de realizar la diagramación del proceso se validó el flujo del proceso según las configuraciones en las reglas de decisión. Se validó el proceso mediante la configuración del comportamiento de las compuertas. Se configuró tiempos estimados de acuerdo al SLA para la ejecución de cada tarea y también se asignó recursos humanos y calendarios para el desarrollo de las diferentes tareas del proceso. Finalmente se simulo 
el proceso lo cual permitió validar en los diferentes escenarios de acuerdo a las configuraciones descritas.

4. Resultados de la Simulación: a partir de los datos obtenidos de la validación y simulación del proceso PGAT se pudo conocer que el modelo propuesto disminuía el tiempo de asistencia técnica en relación al tiempo en el que se atendió las mismas solicitudes sin un proceso establecido.

\section{Resultados}

\section{Análisis de Asistencia Técnica del departamento de TIC's.}

La información analizada corresponde al periodo entre enero 2018 a diciembre de 2018. El número de solicitudes es de 2.777 las cuales se clasifican según: Conectividad, Hardware, Ofimática, Software y varios, como se puede ver en la figura 1.

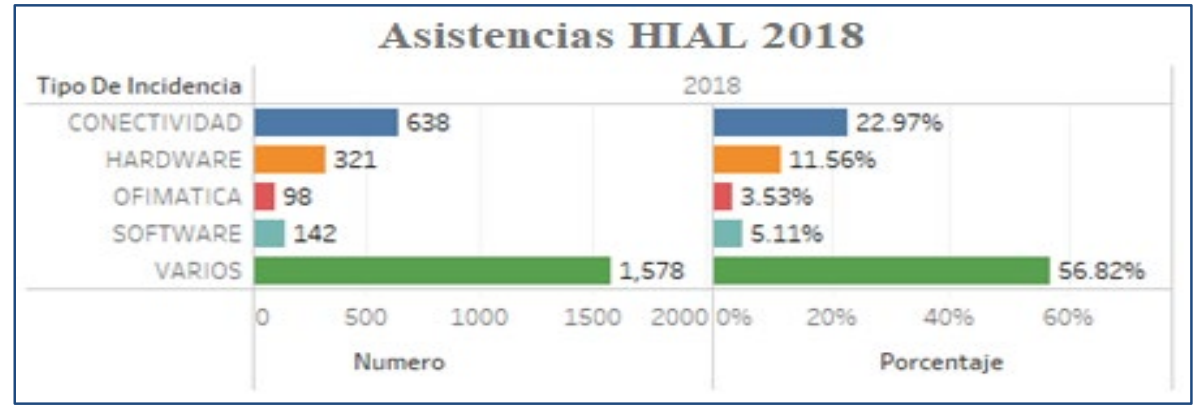

Figura 1: Incidencias HIAL 2018.

Fuente: Elaboración propia.

Para resolver este tipo de solicitudes el personal recepta las ordenes mediante: E-mail, Quipux, Teléfono y personalmente. Se pudo determinar que en un $52,14 \%$ el personal operativo y administrativo reporta personalmente las solicitudes, el 43,82\% lo hace mediante extensión telefónica, el 3,20\% lo realiza mediante Quipux y el 0,83\% mediante el correo institucional al departamento de TIC's. Teniendo como resultado 2777 asistencias que equivalen al 100\% del 2018. 


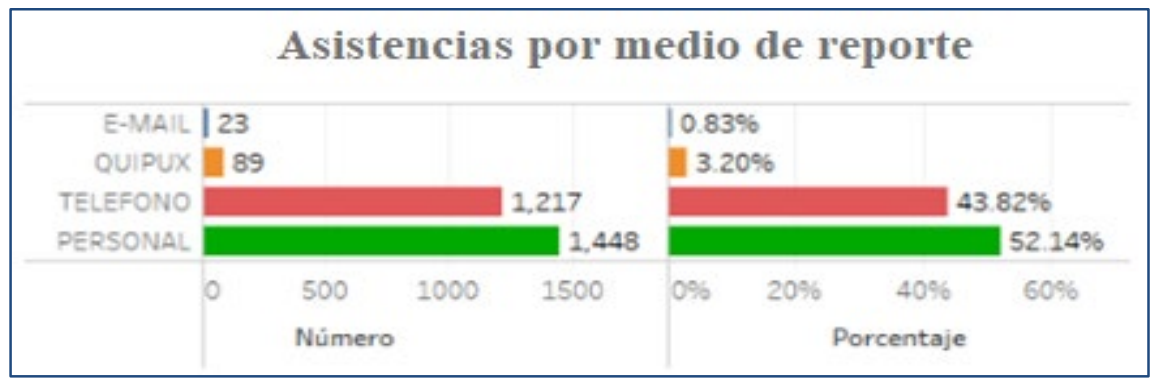

Figura 2: Medio de reporte para asistencias Fuente: Elaboración propia

Para resolver y darles soporte, en el departamento de TIC's se las clasificó de acuerdo a la urgencia y el impacto quedando de la siguiente manera: Urgente, Alto, Medio y Bajo como se puede ver en la tabla 2 .

Tabla 2: Incidencias reportadas por nivel de servicio.

\begin{tabular}{|l|r|r|r|r|r|}
\hline $\begin{array}{l}\text { Nivel de } \\
\text { servicio }\end{array}$ & Cantidad & Tiempo Total & Tiempo Promedio & Total en Días & Porcentaje \\
\hline Urgente & 107 & $224: 34: 00$ & $2: 05: 56$ & 28 & $3,90 \%$ \\
\hline Alto & 259 & $421: 52: 00$ & $1: 37: 44$ & 52 & $9,30 \%$ \\
\hline Medio & 1.882 & $1.473: 04: 00$ & $0: 46: 58$ & 184 & $67,80 \%$ \\
\hline Bajo & 529 & $245: 00: 00$ & $0: 27: 47$ & 30 & $19,00 \%$ \\
\hline Total & $\mathbf{2 . 7 7 7}$ & $\mathbf{2 . 3 6 4 : ~ 3 0 : 0 0}$ & $\mathbf{4 : 5 8 : ~ 2 4}$ & $\mathbf{2 9 5}$ & $\mathbf{1 0 0 \%}$ \\
\hline
\end{tabular}

Fuente: Elaboración propia

Se pudo evidenciar que el 3.90\% de solicitudes se registró como urgente, el 9,30\% como alto, el $67,80 \%$ se registró como medio y el 19,00\% como bajo. El tiempo empleado para resolver las 2.777 solicitudes es de 2.364 horas y 30 minutos. El tiempo promedio de resolución para cada nivel de servicio es de: 2 horas y 5 minutos para urgente, 1 horas con 37 minutos para alto, 46 minutos para medio y 27 minutos para bajo. Se puede evidenciar que la media de los tiempos de resolución para cada tipo de servicio es considerablemente alta, y que el nivel de servicio no se encuentra categorizado de acuerdo al SLA en el cual basa las actividades de gestión de asistencia técnica el departamento de TIC's.

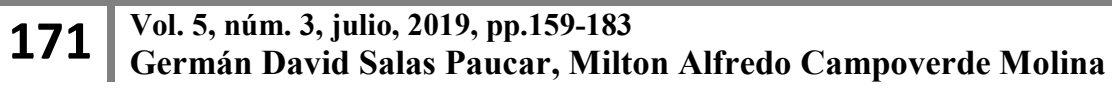




\section{Modelado del proceso de Gestión de Asistencia Técnica (PGAT).}

De acuerdo al análisis de gestión de asistencia técnica que brinda el departamento de TIC's y en base al SLA, se propone la diagramación del proceso denominado "Procesos de Gestión de Asistencia Técnica (PGAT)” en BPMN. 
Dom. Cien., ISSN: 2477-8818

Vol. 5, núm. 3, julio, 2019, pp. 159-183

Proceso de Gestión de Asistencia Técnica. Caso de estudio: Hospital General Isidro Ayora de Loja, Ecuador

Figura 3: Diseño BPM Gestión de Asistencia Técnica.

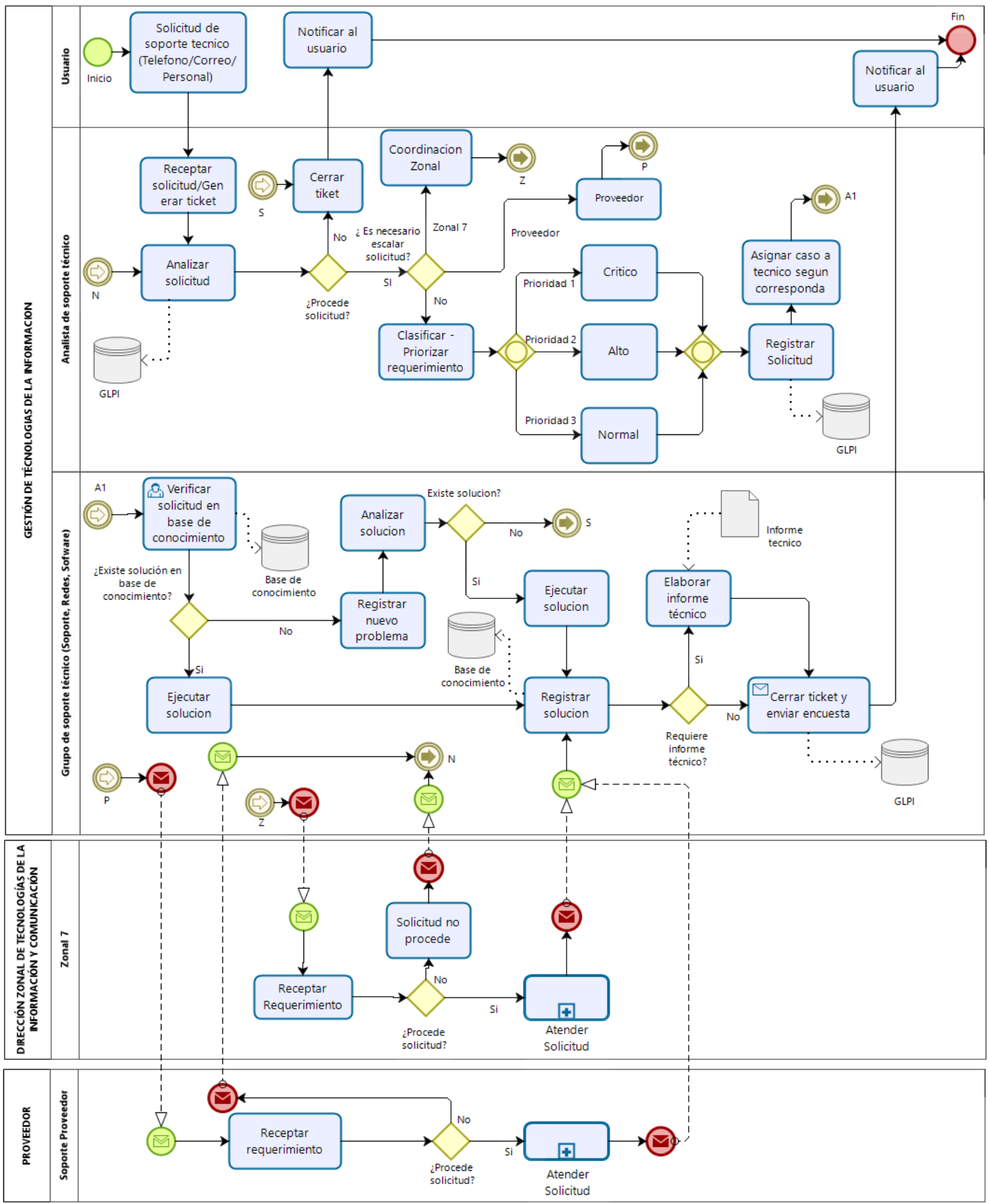

Fuente: Elaboración propia

\section{\begin{tabular}{|l|l|}
173 & Vol. 5, núm. 3, julio, 2019, pp.159-183 \\
Germán David Salas Paucar, Milton Alfredo Campoverde Molina
\end{tabular}}


Dom. Cien., ISSN: 2477-8818

Vol. 5, núm. 3, julio, 2019, pp. 159-183

Proceso de Gestión de Asistencia Técnica. Caso de estudio: Hospital General Isidro Ayora de Loja, Ecuador

\section{Tabla 3: Caracterización de PGAT.}

\begin{tabular}{|c|c|c|}
\hline Macro proceso: & \multicolumn{2}{|c|}{ Tecnologías de la Información y Comunicaciones } \\
\hline Proceso: & \multicolumn{2}{|c|}{ Gestión de Asistencia Técnica } \\
\hline Objetivo & \multicolumn{2}{|c|}{$\begin{array}{l}\text { Identificar reportes de asistencias emitidas por los usuarios internos en } \\
\text { función del soporte de los servicios que brinda el Departamento de } \\
\text { Tecnologías de la Información y Comunicaciones, además de clasificar } \\
\text { dichas incidencias y determinar medios de solución. }\end{array}$} \\
\hline Alcance & \multicolumn{2}{|c|}{$\begin{array}{l}\text { - Desde: Reporte por parte del usuario. } \\
\text { - Hasta: Registro de asistencia, clasificación de asistencia, solución. }\end{array}$} \\
\hline Proveedores & \multicolumn{2}{|c|}{$\begin{array}{l}\text { - Usuario interno Operativo } \\
\text { - Usuario interno Administrativo }\end{array}$} \\
\hline Disparador & \multicolumn{2}{|c|}{ Solicitud de asistencia del usuario. } \\
\hline Insumo(s) & \multicolumn{2}{|c|}{$\begin{array}{l}\text { - Solicitud emitida por el usuario detallando la novedad de la asistencia } \\
\text { - Datos necesarios para el registro de la asistencia }\end{array}$} \\
\hline $\begin{array}{l}\text { Producto(s) } \\
\text { /servicio(s) }\end{array}$ & \multicolumn{2}{|c|}{$\begin{array}{l}\text { - Solicitud atendida } \\
\text { - Solicitud solucionada } \\
\text { - Solicitud escalada }\end{array}$} \\
\hline Clientes internos & \multicolumn{2}{|c|}{ Usuarios Internos HIAL } \\
\hline Políticas & \multicolumn{2}{|c|}{$\begin{array}{l}\text { Toda asistencia se registrará con un ticket como lo establece el acuerdo de } \\
\text { nivel de servicios del departamento de TIC's. }\end{array}$} \\
\hline $\begin{array}{l}\text { Controles } \\
\text { (especificaciones } \\
\text { técnicas y legales) }\end{array}$ & \multicolumn{2}{|c|}{$\begin{array}{l}\text { - Política para la Coordinación de Centro de Soporte de Tecnología, } \\
\text { emitida por la DNTIC(Ministerio de Salud Publica, 2015b). } \\
\text { - Acuerdo de Nivel de Servicios del departamento de TIC's (Hospital } \\
\text { General Isidro Ayora, 2017). } \\
\text { - Normas de control interno para las entidades, organismos del sector } \\
\text { público y de las personas jurídicas de derecho privado que dispongan de } \\
\text { recursos públicos (Contraloría General del Estado, 2009). } \\
\text { - Estatuto orgánico de gestión organizacional por procesos de los } \\
\text { hospitales(Ministerio de Salud Pública, 2012). }\end{array}$} \\
\hline \multirow{4}{*}{ Recursos } & Talento Humano & $\begin{array}{l}\text { - Analista de soporte técnico } \\
\text { - Grupo de soporte técnico }\end{array}$ \\
\hline & Materiales & $\begin{array}{l}\text { - Herramientas hardware y software } \\
\text { - Varios }\end{array}$ \\
\hline & Tecnológicos & $\begin{array}{l}\text { - Equipos informáticos } \\
\text { - Base de conocimiento }\end{array}$ \\
\hline & Financieros & - De acuerdo al presupuesto asignado \\
\hline Frecuencia & \multicolumn{2}{|c|}{ Días Laborables (Lunes a Viernes), 8 horas diarias } \\
\hline
\end{tabular}

Fuente: Elaboración propia

\section{Validación de PGAT.}

Con el fin de garantizar el desempeño eficiente de recurso y un efectivo logro de resultados y antes de una posterior implementación, se procedió a realizar la simulación del proceso en la herramienta 
Bizagi. Se evaluaron 2.777 asistencias brindadas de enero a diciembre del 2018 y de acuerdo al análisis de las asistencias, se procedió a determinar tiempos promedios de solución a cada nivel de asistencia. Además, se estimó el tiempo que llevaría realizar cada una de las tareas y actividades modeladas en el proceso.

Validación de flujo. - Luego de realizar el modelado del proceso PGAT se procedió a validarlo según las configuraciones en las reglas de decisión, por ejemplo, en la clasificación de la solicitud de acuerdo al nivel de servicio ver figura 4 y en si es necesario escalar la solicitud a otro nivel de atención ver figura 5 .

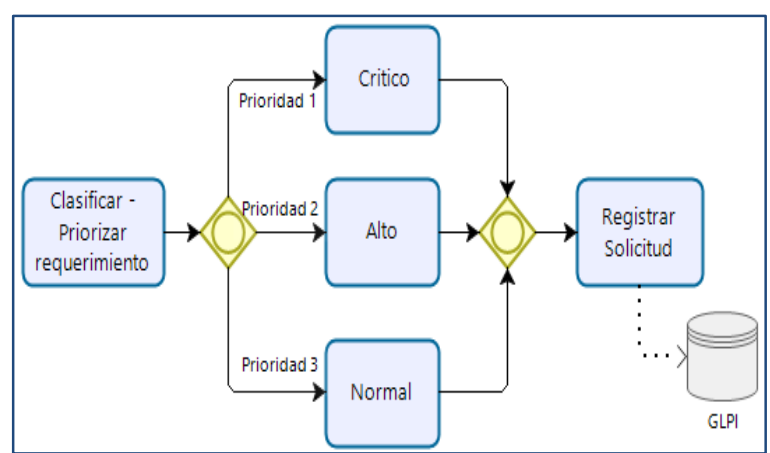

Figura 4: Validación de regla "Clasificación de servicio". Fuente: Elaboración propia.

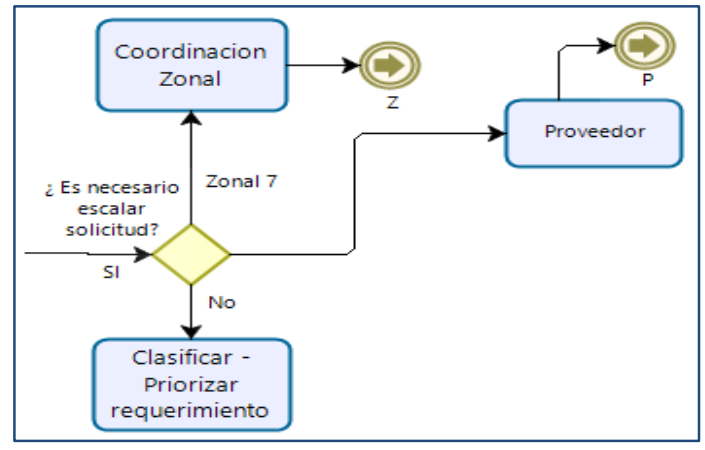

Figura 5: Validación de regla "Escalar solicitud". Fuente: Elaboración propia.

Validación de proceso. - Primero se programó las compuertas de decisión con probabilidades de acuerdo al análisis de asistencias brindadas, y articulado al SLA con el que trabaja el departamento. Así, en la figura 6 se muestra el comportamiento de la compuerta de acuerdo a la configuración quedando de la siguiente manera: Prioridad 1 que equivale a las asistencias registradas como urgentes es de $8 \%$, Prioridad 2 registrado como Alto equivale al 13\% y prioridad 3 que equivale a medio y bajo según las asistencias analizadas quedo con el $79 \%$.

Análisis de tiempo. - Como segundo paso se estableció el tiempo para la ejecución de cada tarea, tiempos estimados de acuerdo a resolución de tareas en las TIC's. Así, en la figura 7 para la tarea "Verificar solicitud en base de conocimiento" se le asignó un tiempo de 2 minutos promedios para desarrollar esta actividad. 


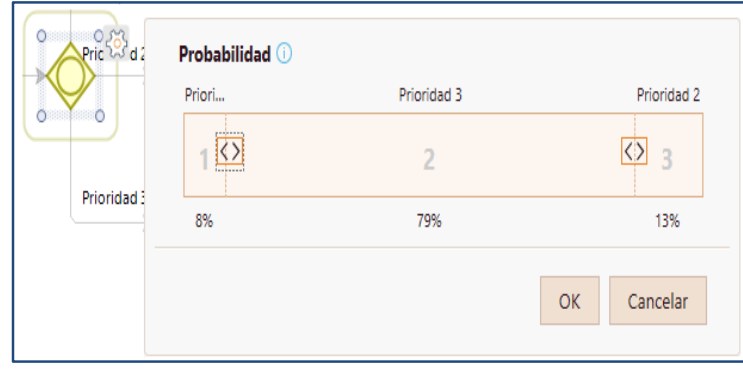

Figura 6: Paso 1, Validación, compuertas divergentes Fuente: Elaboración propia

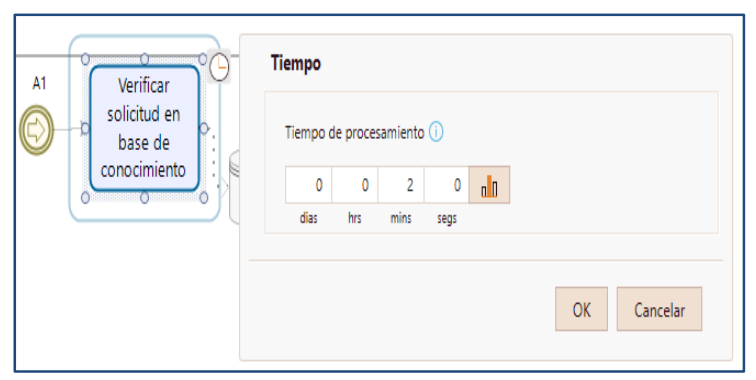

Figura 7: Paso 2, Asignación de tiempos a tareas Fuente: Elaboración propia

Análisis de recursos. - En este paso se asignó los recursos necesarios para cada tarea. En la figura 8 podemos observar que para la tarea "Receptar solicitud/Generar ticket" se asigna 1 "Analista de soporte".

Análisis de calendarios. - Aquí se estableció los días laborables y el horario en el cual desempeña sus actividades el departamento de TIC's durante todo el año. En la figura 9 se observa que el "Analista de soporte técnico" tiene un turno de 8 horas que inicia a las 8 de la mañana de lunes a viernes.

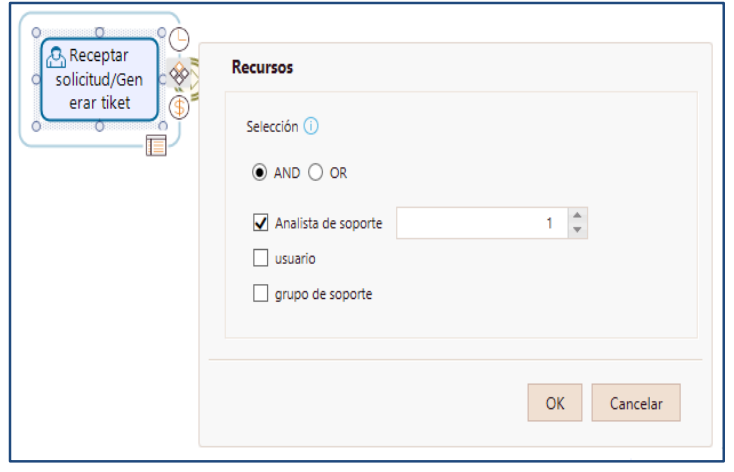

Figura 8: Paso 3, Asignación de recursos.

Figura 9: Paso 4, Creación de calendarios.

Fuente: Elaboración propia.

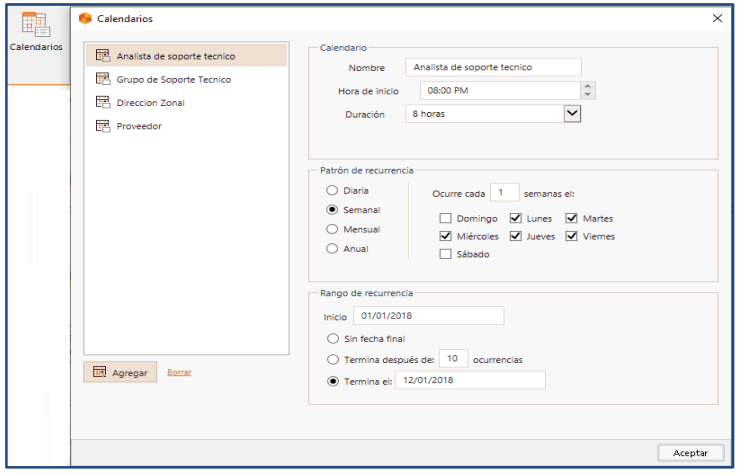

Fuente: Elaboración propia.

Simulación. - En esta etapa para validar la solución generada y con las configuraciones descritas se procedió a realizar la simulación del proceso PGAT, lo cual permitió en los diferentes escenarios 
Dom. Cien., ISSN: 2477-8818

Vol. 5, núm. 3, julio, 2019, pp. 159-183

Proceso de Gestión de Asistencia Técnica. Caso de estudio: Hospital General Isidro Ayora de Loja, Ecuador

realizar la validación de procesos y análisis de tiempo en Bizagi Modeler como se muestra en la figura 10 .

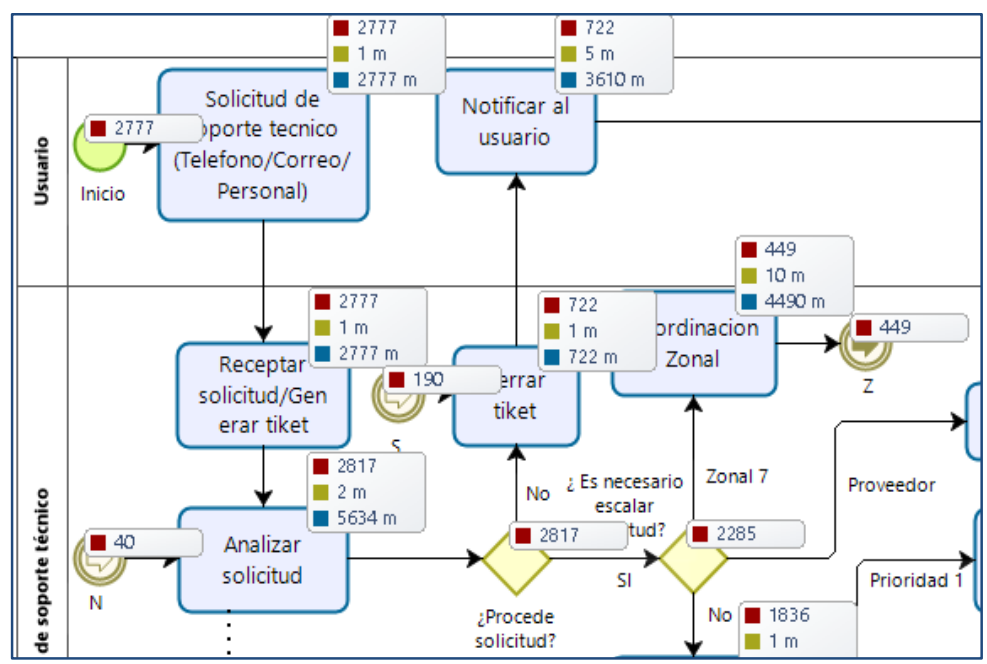

Figura 10: Fragmento de simulación del proceso PGAT.

Fuente: Elaboración propia.

\section{Resultados de simulación}

Se muestra los resultados obtenidos al ejecutar la simulación la cual permitió validar el proceso PGAT. En la tabla 4 se observa que la ejecución fue la esperada ya que de las 2.777 instancias creadas 2.777 fueron ejecutadas satisfactoriamente las cuales se encaminan a las diferentes estancias según las configuraciones definidas en el modelado del proceso y de acuerdo a las probabilidades de que ocurra cada suceso.

Tabla 4: Resultados de simulación, validación de proceso PGAT.

\begin{tabular}{|l|l|r|}
\hline Nombre & Tipo & $\begin{array}{r}\text { Instancias } \\
\text { completadas }\end{array}$ \\
\hline GESTIÓN DE TÉCNOLOGIAS DE LA INFORMACION & Proceso & 2.926 \\
\hline Analizar solicitud & Tarea & 2.791 \\
\hline ¿Procede solicitud? & Compuerta & 2.791 \\
\hline Cerrar ticket & Tarea & 276 \\
\hline Registrar Solicitud & Tarea & 2.384 \\
\hline A1 & Evento intermedio & 2.384 \\
\hline A1 & Evento intermedio & 2.384 \\
\hline ¿Existe solución en base de conocimiento? & Compuerta & 2.384 \\
\hline Ejecutar solución & Tarea & 1.886 \\
\hline
\end{tabular}

\begin{tabular}{|l|l}
177 & Vol. 5, núm. 3, julio, 2019, pp.159-183 \\
Germán David Salas Paucar, Milton Alfredo Campoverde Molina
\end{tabular} 
Dom. Cien., ISSN: 2477-8818

Vol. 5, núm. 3, julio, 2019, pp. 159-183

Proceso de Gestión de Asistencia Técnica. Caso de estudio: Hospital General Isidro Ayora de Loja, Ecuador

\begin{tabular}{|c|c|c|}
\hline Registrar nuevo problema & Tarea & 498 \\
\hline Asignar caso a técnico según corresponda & Tarea & 2.384 \\
\hline Clasificar - Priorizar requerimiento & Tarea & 2.384 \\
\hline Registrar solución & Tarea & 2.501 \\
\hline Notificar al usuario & Tarea & 276 \\
\hline Inicio & Evento de inicio & 2.777 \\
\hline Requiere informe técnico? & Compuerta & 2.501 \\
\hline Elaborar informe técnico & Tarea & 534 \\
\hline Cerrar ticket y enviar encuesta & Tarea & 2.501 \\
\hline Fin & Evento de Fin & 2.777 \\
\hline ¿Es necesario escalar solicitud? & Compuerta & 2.533 \\
\hline Coordinación Zonal & Tarea & 123 \\
\hline Solicitud de soporte técnico(Teléfono/Correo/Personal) & Tarea & 2.777 \\
\hline $\mathrm{Z}$ & Evento intermedio & 123 \\
\hline $\mathrm{N}$ & Evento intermedio & 14 \\
\hline S & Evento intermedio & 18 \\
\hline Z & Evento intermedio & 123 \\
\hline MessageStart & Evento de inicio & 135 \\
\hline MessageStart & Evento de inicio & 8 \\
\hline Notificar al usuario & Tarea & 2.501 \\
\hline Existe solución? & Compuerta & 498 \\
\hline MessageEnd & Evento de Fin & 123 \\
\hline Analizar solución & Tarea & 498 \\
\hline InclusiveGateway & Compuerta & 2.384 \\
\hline Critico & Tarea & 219 \\
\hline Alto & Tarea & 787 \\
\hline Normal & Tarea & 1.378 \\
\hline InclusiveGateway & Compuerta & 2.384 \\
\hline S & Evento intermedio & 18 \\
\hline Ejecutar solución & Tarea & 480 \\
\hline Proveedor & Tarea & 26 \\
\hline $\mathrm{P}$ & Evento intermedio & 26 \\
\hline MessageEnd & Evento de Fin & 26 \\
\hline $\mathrm{P}$ & Evento intermedio & 26 \\
\hline $\mathrm{N}$ & Evento intermedio & 14 \\
\hline MessageStart & Evento de inicio & 6 \\
\hline Verificar solicitud en base de conocimiento & Tarea & 2.384 \\
\hline Receptar solicitud/Generar ticket & Tarea & 2.777 \\
\hline
\end{tabular}

Fuente: Elaboración propia

En la tabla 5 se puede ver los resultados obtenidos del análisis del tiempo. Estos nos permiten tener una idea general del tiempo que toma ejecutar este proceso. En esta simulación se define el tiempo que un usuario tiene que esperar para que una solicitud de asistencia técnica sea atendida y resuelta.

\footnotetext{
$178 \begin{aligned} & \text { Vol. 5, núm. 3, julio, 2019, pp.159-183 } \\ & \text { Germán David Salas Paucar, Milton Alfredo Campoverde Molina }\end{aligned}$
} 
Dom. Cien., ISSN: 2477-8818

Vol. 5, núm. 3, julio, 2019, pp. 159-183

Proceso de Gestión de Asistencia Técnica. Caso de estudio: Hospital General Isidro Ayora de Loja, Ecuador

Para este caso se simulo un total de 2.777 solicitudes de asistencia técnica registradas durante el 2018. Se puede observar que por ejemplo una solicitud de asistencia técnica brindada por el departamento de TIC's sin necesidad de escalar a otro nivel de atención tarda en promedio 23 minutos con 9 segundos en ser atendida, una solicitud escalada a la Dirección Zonal de Tecnologías de la Información y Comunicación tarda 38 minutos con 10 segundos en ser atendida y la atención escalada al proveedor tarda en promedio 51 minutos con 20 segundos en ser atendida.

Tabla 5: Resultados de simulación, validación de proceso PGAT.

\begin{tabular}{|c|c|c|c|c|c|c|c|}
\hline Nombre & $\stackrel{\circ}{\stackrel{2}{2}}$ & 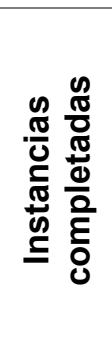 & 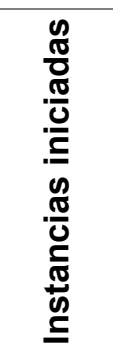 & 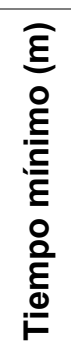 & 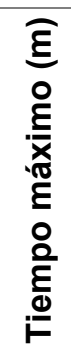 & 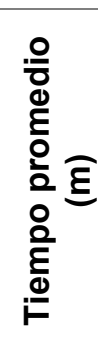 & 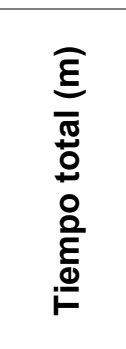 \\
\hline $\begin{array}{l}\text { Gestión de Tecnologías de la } \\
\text { Información HIAL }\end{array}$ & Proceso & 2.926 & 2.926 & 3 & 37 & 23,9 & 70.024 \\
\hline Analizar solicitud & Tarea & 2.791 & 2.791 & 2 & 2 & 2 & 5.582 \\
\hline Cerrar ticket & Tarea & 276 & 276 & 1 & 1 & 1 & 276 \\
\hline Registrar Solicitud & Tarea & 2.384 & 2.384 & 1 & 1 & 1 & 2.384 \\
\hline Ejecutar solución & Tarea & 1.886 & 1.886 & 15 & 15 & 15 & 28.290 \\
\hline Registrar nuevo problema & Tarea & 498 & 498 & 1 & 1 & 1 & 498 \\
\hline Asignar caso a técnico según corresponda & Tarea & 2.384 & 2.384 & 0,5 & 0,5 & 0,5 & 1.192 \\
\hline Clasificar - Priorizar requerimiento & Tarea & 2.384 & 2.384 & 1 & 1 & 1 & 2.384 \\
\hline Registrar solución & Tarea & 2.501 & 2.501 & 1 & 1 & 1 & 2.501 \\
\hline Notificar al usuario & Tarea & 276 & 276 & 1 & 1 & 1 & 276 \\
\hline Elaborar informe técnico & Tarea & 534 & 534 & 5 & 5 & 5 & 2.670 \\
\hline Cerrar ticket y enviar encuesta & Tarea & 2.501 & 2.501 & 1 & 1 & 1 & 2.501 \\
\hline Coordinación Zonal & Tarea & 123 & 123 & 1 & 1 & 1 & 123 \\
\hline $\begin{array}{l}\text { Solicitud de soporte } \\
\text { técnico(Teléfono/Correo/Personal) }\end{array}$ & Tarea & 2.777 & 2.777 & 1 & 1 & 1 & 2.777 \\
\hline Notificar al usuario & Tarea & 2.501 & 2.501 & 1 & 1 & 1 & 2.501 \\
\hline Analizar solución & Tarea & 498 & 498 & 5 & 5 & 5 & 2.490 \\
\hline Critico & Tarea & 219 & 219 & 0,5 & 0,5 & 0,5 & 109,5 \\
\hline Alto & Tarea & 787 & 787 & 0,5 & 0,5 & 0,5 & 393,5 \\
\hline Normal & Tarea & 1.378 & 1.378 & 0,5 & 0,5 & 0,5 & 689 \\
\hline Ejecutar solución & Tarea & 480 & 480 & 15 & 15 & 15 & 7200 \\
\hline Proveedor & Tarea & 26 & 26 & 1 & 1 & 1 & 26 \\
\hline Verificar solicitud en base de conocimiento & Tarea & 2.384 & 2.384 & 1 & 1 & 1 & 2.384 \\
\hline Receptar solicitud/Generar ticket & Tarea & 2.777 & 2.777 & 1 & 1 & 1 & 2.777 \\
\hline Tiempo en días & & & & & & & 145,8 \\
\hline
\end{tabular}


Dom. Cien., ISSN: 2477-8818

Vol. 5, núm. 3, julio, 2019, pp. 159-183

Proceso de Gestión de Asistencia Técnica. Caso de estudio: Hospital General Isidro Ayora de Loja, Ecuador

\begin{tabular}{|l|l|r|r|r|r|r|r|}
\hline $\begin{array}{l}\text { Dirección Zonal de Tecnologías de la } \\
\text { Información y Comunicación }\end{array}$ & Proceso & $\mathbf{1 2 3}$ & $\mathbf{1 2 3}$ & $\mathbf{1 1}$ & $\mathbf{4 0}$ & $\mathbf{3 8 , 1}$ & $\mathbf{4 . 6 8 8}$ \\
\hline Receptar Requerimiento & Tarea & 123 & 123 & 10 & 10 & 10 & 1.230 \\
\hline Solicitud no procede & Tarea & 8 & 8 & 1 & 1 & 1 & 8 \\
\hline Atender Solicitud & Tarea & 115 & 115 & 30 & 30 & 30 & 3.450 \\
\hline Tiempo en días & & & & & & & $\mathbf{9 , 7}$ \\
\hline Proveedor & & & & & & & $\mathbf{6 3}$ \\
\hline Receptar requerimiento & Proceso & $\mathbf{2 6}$ & $\mathbf{2 6}$ & $\mathbf{5}$ & $\mathbf{6 5}$ & $\mathbf{5 1 , 2}$ & $\mathbf{1 . 3 3 0}$ \\
\hline Atender Solicitud & Tarea & 26 & 26 & 5 & 5 & 5 & 130 \\
\hline Tiempo en días & Tarea & 20 & 20 & 60 & 60 & 60 & 1.200 \\
\hline
\end{tabular}

Fuente: Elaboración propia

Por último, se calculó el índice de eficiencia del proceso PGAT (ver figura 4). Por consiguiente, para atender las 2.777 solicitudes de asistencia técnica reportadas durante el 2018 se necesitó un total de 158 días y 4 horas un $46.3 \%$ menos de tiempo en relación al tiempo en el que se atendió las mismas solicitudes sin un proceso establecido el cual fue de 295 días (ver tabla 2).

\section{Conclusiones}

La gestión de asistencias técnicas en el Hospital Isidro Ayora de Loja, es el resultado de la interacción entre el departamento de TIC's y los usuarios de las diferentes áreas. En este contexto es de vital importancia el aporte realizado en este trabajo de investigación, ya que los resultados obtenidos del análisis, modelado, validación y simulación del "Proceso de Gestión de Asistencia Técnica" (PGAT), ayudó a determinar que la automatización de este proceso reduciría en un 46,3\% el tiempo empleado en resolver las solicitudes de asistencia generadas por los usuarios.

Un aspecto importante de este trabajo de investigación es que analizar las actividades que desarrolla el departamento de TIC's, permitió obtener la información necesaria para el levantamiento del proceso. Además, la utilización de Bizagi Modeler para modelar y simular el proceso siguiendo la notación BPMN facilito las tareas de diseñar, modelar y simular el proceso y determinar que es posible mejorar sustancialmente el grado de cumplimiento de la gestión de asistencia técnica que se brinda a los usuarios. 
De acuerdo a los resultados obtenidos en esta investigación, se pudo determinar la necesidad de definir procesos que permitan optimizar las actividades de los diferentes servicios y así lograr alinearlas de mejor manera con los objetivos estratégicos de la institución.

\section{Referencias Bibliográficas}

Bizagi. (2003). Modelado para ejecución Bizagi. Retrieved June 13, 2019, from http://help.bizagi.com/bpm-suite/es/index.html?bpmn_shapes.htm

Bizagi. (2014). Bizagi BPM Suite, 36. Retrieved from http://resources.bizagi.com/docs/Bizagi Descripcion Funcional.pdf

Bizagi. (2016). Guía de Usuario de Bizagi Process Modeler - Una Heramienta de Modelamiento de Procesos de Negocio. Retrieved June 13, 2019, from http://help.bizagi.com/process-modeler/es/ Braun, R., Burwitz, M., Schlieter, H., \& Benedict, M. (2015). Clinical processes from various angles - amplifying BPMN for integrated hospital management. In 2015 IEEE International Conference on Bioinformatics and Biomedicine (BIBM) (pp. 837-845). IEEE. https://doi.org/10.1109/BIBM.2015.7359794

Congacha, A., \& Garcia, V. (2017). Modelación, simulación y automatización de procesos en la gestión de servicios académicos universitarios. 3c Tecnología: Glosas de Innovación Aplicadas a La Pyme, ISSN-e 2254-4143, Vol. 6, No. 2, 2017, Págs. 32-51, 6(2), 32-51. Retrieved from https://dialnet.unirioja.es/servlet/articulo?codigo $=6034899$

Contraloría General del Estado. (2009). NORMAS DE CONTROL INTERNO PARA LAS ENTIDADES, ORGANISMOS DEL SECTOR PÚBLICO Y DE LAS PERSONAS JURÍDICAS DE DERECHO PRIVADO QUE DISPONGAN DE RECURSOS PÚBLICOS, 1-86. Retrieved from http://ai.espe.edu.ec/wp-content/uploads/2012/07/ACUERDO-039-CG-5-Normas-deControl-Interno.pdf

EcuRed. (2018). Organización Mundial de la Salud. Retrieved June 5, 2019, from https://www.ecured.cu/Organización_Mundial_de_la_Salud 
Garimella, K., Michael Lees, \& Bruce Williams. (2012). BPM (GERENCIA DE PROCESOS DE NEGOCIO) Tomado del Libro BPM. GERENCIA DE PROCESOS DE NEGOCIO.

Hernández, D., Pérez, D., León, E., Infante, M., \& Blanco, J. (2015). Propuesta de proceso de planificación de sistemas de información para la industria biofarmacéutica cubana Proposal of an information systems planning procedure for the Cuban biopharmaceutical industry, 27(2), 185205. Retrieved from http://scielo.sld.cu

Hospital General Isidro Ayora. (2017). Acuerdo de Nivel de Servicios (SLA), 1-16.

Laurentiis, G. (2011). Metodología BPM: RAD®-Rapid Analysis \& Design para la modelización y diseño de procesos orientados a tecnologías BPM. En El Libro Del BPM: Tecnologías, Conceptos, $\quad$.., 115-136. $\quad$ Retrieved from http://scholar.google.com/scholar?hl=en\&btnG=Search\&q=intitle:Metodolog?a+BPM+:+RAD+? + + +Rapid + Analysis $+\&+$ Design + para + la + modelizaci? $n+y+$ dise $o+d e+$ procesos + orientados $+\mathrm{a}+$ te cnolog?as+BPM\#0

Leon, C. A. M. (2010). Programas De Producción Hospitalaria Y Herramienta De.

Marc Kerremans, Derek Miers, Rob Dunie, Jason Wong, Kimihiko Iijima, P. V. (2019). Magic Quadrant for Intelligent Business Process Management Suites. Retrieved June 17, 2019, from https://www.gartner.com/en/documents/3899484

Martinho, R., Rijo, R., \& Nunes, A. (2015). Complexity Analysis of a Business Process Automation: Case Study on a Healthcare Organization. Procedia Computer Science, 64, 12261231. https://doi.org/10.1016/j.procs.2015.08.510

Ministerio de Salud Publica. Coordinación de Centro de Soporte de Tecnología (2012). Retrieved from

http://instituciones.msp.gob.ec/images/Documentos/Ministerio/tic/Politica_Coordinacion_Centro _Soporte_Tecnologia.pdf

Ministerio de Salud Publica. (2012b). ESTATUTO ORGÁNICO DE GESTIÓN ORGANIZACIONAL POR PROCESOS DE LOS HOSPITALES. Msp, 1-50. Retrieved from 
http:/instituciones.msp.gob.ec/somossalud/images/documentos/guia/Estatuto_hospitales_MOD.p $\mathrm{df}$

Ministerio de Salud Publica. (2012c). Ministerio de Salud Publica> Planificación, gestión, coordinación y control de la salud pública. Retrieved June 6, 2019, from https://www.salud.gob.ec/el-ministerio/

Ministerio de Salud Publica. (2012d). Ministerio de Salud Pública - El Ministerio de Salud Pública ejerce la rectoría del Sistema Nacional de Salud a fin de garantizar el derecho a la salud del pueblo ecuatoriano. Retrieved June 7, 2019, from https://www.salud.gob.ec/

Ministerio de Salud Publica. (2015a). Coordinación Zonal de Salud 7 rinde cuentas a la ciudadanía - Ministerio de Salud Pública. Retrieved June 10, 2019, from https://www.salud.gob.ec/coordinacion-zonal-de-salud-7-rinde-cuentas-a-la-ciudadania/

Ministerio de Salud Publica. (2015b). Dirección Nacional de Tecnologías de la Información y Comunicaciones - Ministerio de Salud Pública. Retrieved June 6, 2019, from https://www.salud.gob.ec/direccion-nacional-de-tecnologias-de-la-informacion-ycomunicaciones/

Ministerio de Salud Publica. Manual de Procesos operativos para Hospitales Generales del MSP (2016). Ecuador.

Organización Panamericana de la Salud. (2014). Organización Panamericana de la Salud (OPS). Retrieved June 5, 2019, from https://www.paho.org/hq/index.php?option=com_content\&view=article\&id=91:aboutpaho\&Itemid $=220 \& l a n g=e s$

Rolón, E., Chavira, G., Orozco, J., \& Soto, J. P. (2015). Towards a Framework for Evaluating Usability of Business Process Models with BPMN in Health Sector. Procedia Manufacturing, 3, 5603-5610. https://doi.org/10.1016/j.promfg.2015.07.748 